\title{
Deformation behavior of crosslinked polyurea elastomers obtained via sol-gel chemistry: experimental determination and constitutive modelling
}

\author{
N. Rull ${ }^{1}$, A. Sánchez-Ferrer ${ }^{2}$, P. M. Frontini ${ }^{1}$ \\ ${ }^{1}$ Instituto de Investigación en Ciencia y Tecnología de Materiales INTEMA, CONICET, UNMdP, Av. J. B. Justo 4302, \\ B7608FDQ, Mar del Plata, Argentina \\ ${ }^{2}$ Technical University of Munich (TUM), Wood Research Munich (HFM), Winzererstr. 45, D-80797 Munich, Germany
}

Received 17 October 2019; accepted in revised form 2 December 2019

\begin{abstract}
The mechanical behavior of three crosslinked polyurea (PU) elastomers obtained by sol-gel chemistry under uniaxial monotonic and cyclic tests has been assessed. These elastic networks are composed of hard and soft domains, where the segmental molecular weight between crosslinking points differs among the samples and allow studying the effect of this parameter on the mechanical properties. In this paper, uniaxial tension tests were performed in order to capture the main characteristics of the stress-strain behavior of these PU elastomers, e.g., non-linear hyperelastic behavior, hysteresis, and softening. In addition, a constitutive model that properly represents their behavior was proposed showing that the non-linear stress-strain behavior at large strain values exhibits strong hysteresis and softening.
\end{abstract}

Keywords: mechanical properties, polyurea elastomer, constitutive modeling, softening

\section{Introduction}

When comparing the performance of polyurea (PU) elastomers with their polyurethane and polyester counterparts, it has been found that PU elastomers have better characteristics, such as chemical resistance. This can be attributed to the presence of welldefined hydrogen bonds [1]. The reaction between amines and isocyanates does not require any catalyst, is conducted at room temperature, and is faster than carbamation and esterification reactions. Moreover, due to the presence of inter-chain mono and bi-dentate urea hydrogen bonds, PUs result in double chemical and physical polymer networks showing microphase separated domains comparable to nanocomposite materials: hard domains embedded in a soft polymer matrix [2].

Nowadays, PU materials have found many applications due to their improved properties such as abrasion resistance, corrosion protection, waterproofing, and structural enhancement, making PU a preferred material due to its ease processing - machine and liquid molding, and fiber spinning for tubing, coating, foams, fibers, sheets, and film production - and wide implementation in medical devices and applications - cardiovascular items, reconstructive surgery, gynecology and obstetrics, organ regeneration, tissue engineering, and mechanical supplies [3-6].

PU elastomers are morphologically characterized by the presence of soft and hard domains, which are constituted by the polymer melt (diamino-terminated polymer) and the crosslinker (polyisocyanate), respectively. The interfacial urea groups formed during the reaction establish strong hydrogen bonds [2, 7], which are responsible of physical crosslinking and define the micro-phase separated domains, translating such chemical interactions to macroscopic physical 
properties like enhanced mechanical and chemical resistance, flexibility, toughness, excellent electrical insulating, and good adhesion properties than standard polyurethanes and polyesters [7]. When compared to other hydrogen bonding groups commonly found in segmented copolymers, urea groups impart one of the strongest inter-chain interactions [8].

This paper is concerned with the evaluation of chemically crosslinked PU elastomers, whose chemistry is based on a chemical reaction between the electrophilic isocyanate group and the nucleophilic amine group to form urea motives which establish hydrogen bonds (physical crosslinks). Three PU materials synthesized via sol-gel chemistry are investigated, and they differ from the polymer backbone degree of polymerization (DP) - segmental molecular weight, $M_{\mathrm{c}}$, or segmental length, $l_{\mathrm{c}}-$, which defines the final volume fraction of both soft $\left(\phi_{\mathrm{p}}\right)$ and hard domains $\left(\phi_{c}\right)$. The resulting change in the soft domain concentration controls the viscoelastic or viscoplastic properties of the crosslinked PU material, which is reflected in its mechanical properties and stress-strain behavior.

Microphase separated PU materials based on noncrosslinked linear segmented copolymers have been thoroughly studied using different test configurations at both low and high strain rates [9-11]. Research on simple hyperelastic constitutive models, i.e., Money-Rivlin and Ogden, has also been performed to describe the PU behavior under uniaxial loading [12-14]. The crosslinked PU materials studied in this paper differ from those mentioned above because they are directly dependent on their segmental molecular weight $\left(M_{\mathrm{c}}\right)$. These crosslinked polymer chains cannot slip from one soft domain to another as non-crosslinked PU materials do when stretching the sample at large stress values. Therefore, a difference in the mechanical properties between thermosetting elastomers (our crosslinked PU materials) and thermoplastic elastomers (the common non-crosslinked PU materials) must be observed, and more sophisticated constitutive models [15] have been developed in order to accurately describe the mechanical behavior of such PU materials under various loading conditions.

The mechanical properties of polyurethane/polyurea elastomers as a function of the segmental molecular weight of the soft polymeric component were evaluated $[16,17]$. Both studies showed a significant change in the response of these materials and hysteresis behavior; hence the constitutive modeling of such materials was not straight forward.

In previous scientific works, the mechanical behavior of these crosslinked PUs was evaluated at the maximum strain value of $\varepsilon_{\max }=0.01$ in quasi-static loading conditions $[6,7]$. Currently, we aim to find a constitutive model that properly captures the mechanical behavior of these PU elastomers up to a strain value of $c a . \varepsilon=1$, and at two different strain rates. The ability to predict the mechanical behavior of elastomeric materials is a technological problem still being solved [18] since it is highly dependent on stress conditions such as temperature, frequency, strain, and stress states $[19,20]$. Herein, we present the uniaxial tensile tests performed on such crosslinked networks and the constitutive models capturing the major features of the stress-strain behavior of these crosslinked PU elastomers, including nonlinear hyperelastic behavior, time dependence, hysteresis and softening [21,22].

\section{Experimental}

\subsection{Materials}

Three linear diamino-terminated poly(propylene oxide) Jeffamine ${ }^{\circledR} \mathrm{D}-400\left(M_{\mathrm{n}}=460 \mathrm{Da}, \mathrm{DP} \sim 6\right)$, D$2000\left(M_{\mathrm{n}}=2060 \mathrm{Da}, \mathrm{DP} \sim 34\right)$ and D-4000 $\left(M_{\mathrm{n}}=\right.$ $4000 \mathrm{Da}, \mathrm{DP} \sim 68)$ from Huntsman International LLC (Pétfürdő, Hungary) were reacted with the corresponding stoichiometric amount of the triisocyanate crosslinker Basonat ${ }^{\circledR} \mathrm{HI}-100\left(M_{\mathrm{n}}=505 \mathrm{Da}\right)$ provided by BASF SE (Ludwigshafen, Germany) in acetone (Acros Organics, Schwerte, Germany) at room temperature - details of the synthesis are

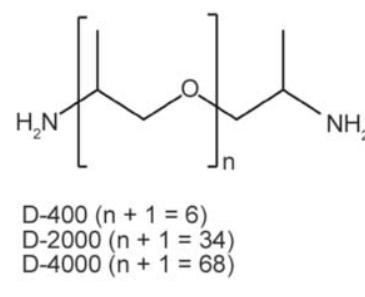

a)

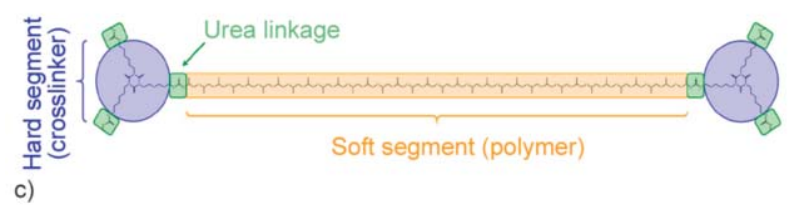

Figure 1. a) Chemical structure of the diamino-terminated poly(propylene oxide) Jeffamine ${ }^{\circledR}$ D-400, D-2000 and D-4000, b) the triisocyanate crosslinker Basonat ${ }^{\circledR}$ HI-100, and c) the resulting network when the urea motives are formed. 
Table 1. Number-average molar mass $\left(M_{\mathrm{n}}\right)$, degree of polymerization (DP), crosslinker volume fraction $\left(\phi_{\mathrm{c}}\right)$, segmental molecular weight $\left(M_{\mathrm{c}}\right)$, segmental length $\left(l_{\mathrm{c}}\right)$, and glass transition temperature $\left(T_{\mathrm{g}}\right)$ for the three PU elastomers.

\begin{tabular}{|l|r|c|c|r|r|c|}
\hline $\begin{array}{c}\text { PU } \\
\text { elastomer }\end{array}$ & $\begin{array}{c}\boldsymbol{M}_{\mathbf{n}} \\
{[\mathbf{D a}]}\end{array}$ & $\mathbf{D P}$ & $\boldsymbol{\phi}_{\mathbf{c}}$ & $\begin{array}{c}\boldsymbol{M}_{\mathbf{c}} \\
{[\mathbf{D a}]}\end{array}$ & $\begin{array}{c}\boldsymbol{l}_{\mathbf{c}} \\
{[\mathbf{n m}]}\end{array}$ & $\begin{array}{c}\boldsymbol{T}_{\mathbf{g}} \\
{\left[{ }^{\circ} \mathbf{C}\right]}\end{array}$ \\
\hline ED-400 & 460 & 6 & 0.39 & 428 & 2.5 & -24 \\
\hline ED-2000 & 2060 & 34 & 0.12 & 1990 & 12.1 & -65 \\
\hline ED-4000 & 4000 & 68 & 0.07 & 4024 & 24.5 & -71 \\
\hline
\end{tabular}

described in the references $[2,7,23,24]$. The chemical structures for the polyetheramines, the crosslinker, and the resulting ED-400, ED-2000 and ED-4000 networks are shown in Figure 1, and the main characteristics of the three PU networks are summarized in Table 1.

\subsection{Specimens and mechanical tests}

Tension test experiments were carried out using dog bone specimens (Figure 2a) with section dimensions of $25 \times 4$ 0.5-0.6 mm and obtained from circular PU elastomeric films of thickness between 0.5 and $0.6 \mathrm{~mm}$ by properly die-cutting. The grip section width and length were $12.5 \times 12.5 \mathrm{~mm}$, respectively. An INSTRON 4340 mechanical machine with displacement control, equipped with a load cell of $0.5 \mathrm{kN}$ and clamps for the configuration selected, were used. In order to accurately measure the strain variation during testing, a video system recorded the sample evolution under deformation with a JAI CCD camera CV-M50 (30 fps) and a NAVITAR TV ZOOM-7000 (Figure 2b). Two circular marks were made on each specimen with a special elastic gel ink.
Distance between marks $l_{0}$ was measured initially and filmed during testing by the camera. Later, the digital analysis allowed obtaining true measures of strain, by comparing distances between markers along time.

These tests were performed at room temperature $\left(\sim 23^{\circ} \mathrm{C}\right)$, at an initial strain rate of $100 \% \cdot \mathrm{min}^{-1}(\dot{\varepsilon}=$ $\left.1 \mathrm{~min}^{-1}\right)$ and $500 \% \cdot \mathrm{min}^{-1}\left(\dot{\varepsilon}=5 \mathrm{~min}^{-1}\right)$, and three tests per each tested condition $(n=3)$. Additionally, one set of samples was thermally treated for $30 \mathrm{~min}$ at $70^{\circ} \mathrm{C}$ in order to promote the rupture of the hydrogen bonds [2]. Afterward, samples were cooled down to room temperature at different cooling rates: i) at the slow rate of $\mathrm{d} T / \mathrm{d} t=0.5^{\circ} \mathrm{C} \cdot \mathrm{min}^{-1}$ for allowing the hydrogen bond reformation, and ii) fastly cooled down to room temperature in order to quench the network configuration. In all cases, samples were tested as soon as room temperature was reached. Specimens were tested up to a maximum strain value of $\varepsilon_{\max }=0.5-0.6$, and three loading-unloading cycles were performed in order to investigate the presence of hysteresis.

Uniaxial stress relaxation tensile test experiments were also conducted for the three PU samples at a cross-head speed of $200 \% \cdot \mathrm{min}^{-1}$. For each PU material and during the loading phase, two stress relaxation steps were imposed at the constant strain values of $\varepsilon=0.2$ and $\varepsilon=0.4$, during $60 \mathrm{~s}$. After the $60 \mathrm{~s}$ of holding at constant strain, samples were loaded at the same speed as shown in Figure 2c.

Data obtained as retractive force $v s$. displacement were converted to true stress $v s$. true strain using the following Equations (1) and (2):

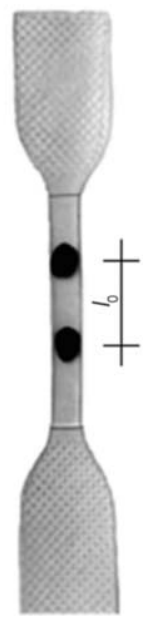

a)

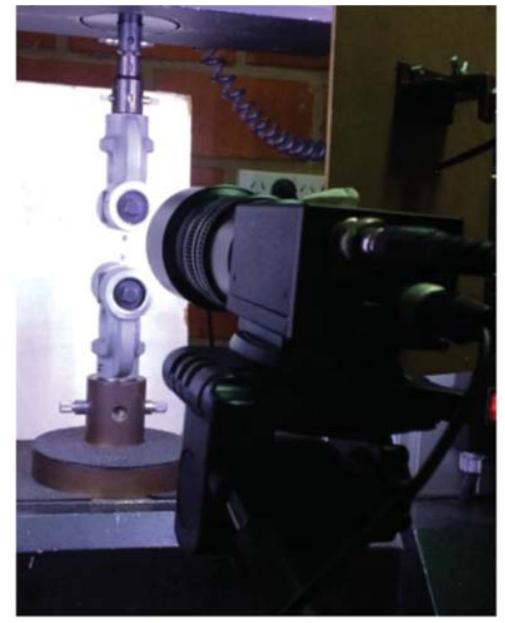

b)

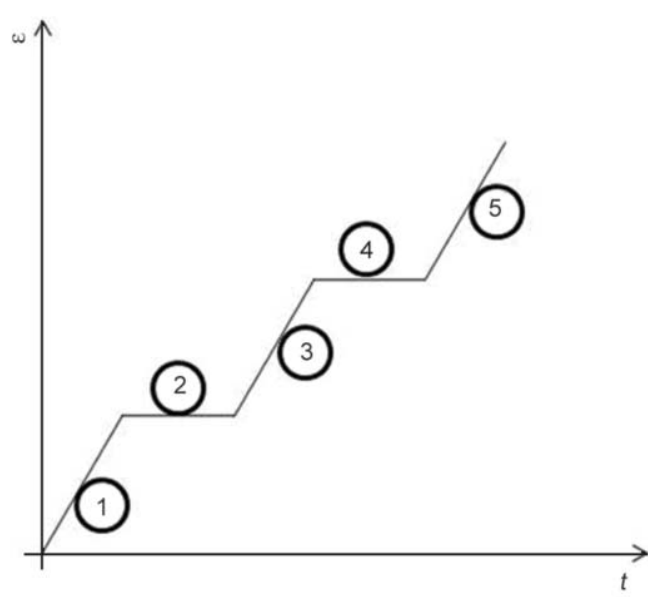

c)

Figure 2. a) Specimen scheme for tensile tests according to ISO standard 37. b) Tension test apparatus with the specimen and camera disposition. c) Schematic representation of the stress relaxation tensile test experiments. Steps 1, 3 and 5 are at constant strain rate; and steps 2 and 4 are at constant strain value for $60 \mathrm{~s}$. 
$\varepsilon_{\text {true }}=\ln \lambda$

$\sigma_{\text {true }}=\sigma \cdot \lambda$

where $\lambda=l / l_{0}, \sigma$ is the measured force per initial area of the sample, $l_{0}$ the initial length between dots and $l$ is the specimen's dots distance at each deformation step.

\section{Results and discussion}

\subsection{Mechanical behavior}

Figure 3 compares the true stress-true strain plots for the three crosslinked PU materials together under two different strain rates (Figure $3 \mathrm{a}$ at $100 \% \cdot \mathrm{min}^{-1}$ and Figure $3 \mathrm{~b}$ at $500 \% \cdot \mathrm{min}^{-1}$ ). Depending on the degree of polymerization (DP) of the material, different behaviors were observed. All experimental data were fitted by using a heuristic formula already used for the mechanical analysis of liquid-crystalline elastomers $[25,26]$ consisting on the contribution of a linear, a positive exponential growth and a negative exponential decay function. The resulting fitting curve and its corresponding derivative allows for the determination of the initial elastic modulus (Young modulus), the minimum elastic modulus (softening behavior), the hysteresis factor between loading and unloading experiments, and the energy loss between cycles.

For both the ED-2000 and ED-4000 samples, in which the volume fraction of hard domains is lower $\left(\phi_{\mathrm{c}}=0.12\right.$ and 0.07 , respectively) than for the ED-400 network $\left(\phi_{\mathrm{c}}=0.39\right)$, a typical elastomeric behavior was observed featuring large deformation and almost full recovery after unloading. The corresponding

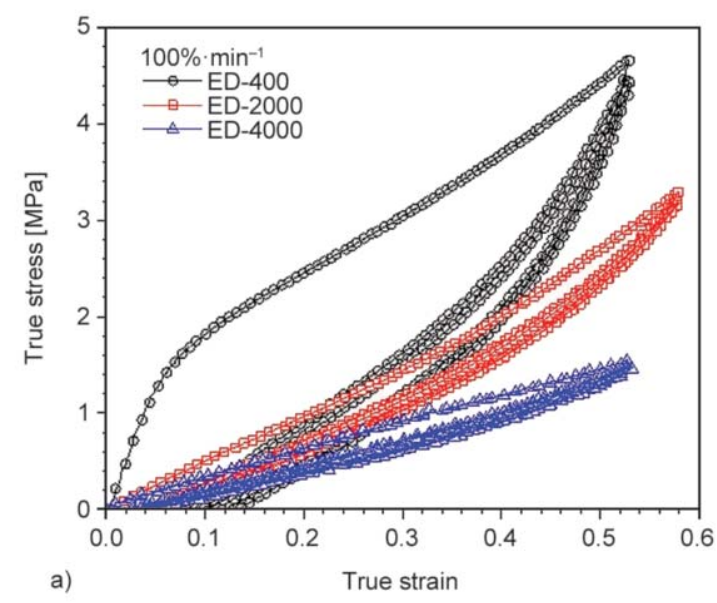

Young modulus was 6 and 3 MPa for the ED-2000 and the ED-4000 samples, respectively, with moderate hysteresis $(\eta \approx 0.75$ for the first cycle, and $\eta \approx$ 0.90 for the second and third one), and energy recovery of $c a .80 \%$ for both samples. On the other hand, sample ED-400 shows a stiff response for the first portion of the loading curve and a yielding behavior after a strain value of about $\varepsilon_{\text {true }}=0.1$. The evaluated Young modulus was $c a .45 \mathrm{MPa}$ for the first cycle while dropping to $c a$. $10 \mathrm{MPa}$ for the following cycles. The unloading path shows a large hysteresis loop ( $\eta \approx 0.45$ for the first cycle, and $\eta \approx 0.80$ for the second and third one), with a residual strain value of ca. $\varepsilon_{\text {true }}=0.1$ and energy recovery of $c a .60 \%$. The unusual initial stiff behavior can be interpreted as the 'glass-like' response with a clear yield event followed by a modest hardening due to the short segmental polymer length, lc, of this sample.

Strain rate dependence tensile experiments were also analyzed for the three materials separately (Figure 4). A small dependence with the strain rate within the strain range analyzed was observed for both the ED2000 and ED-4000 elastomers. On the contrary, for the ED-400 sample, it was perceived that the stress increased at a higher strain rate for the loading portions of the curves. The second cycle of the true stresstrue strain curve shows a decrease in the stress for the same strain value compared to the first cycle (Figure 4). This observation can be related to material softening. In addition, the stress-strain behavior tends to stabilize after the first cycle, showing no significant difference between the second and third cycles. The behavior found is similar to that observed by Qi and Boyce [27] in which the polyurethane samples tested

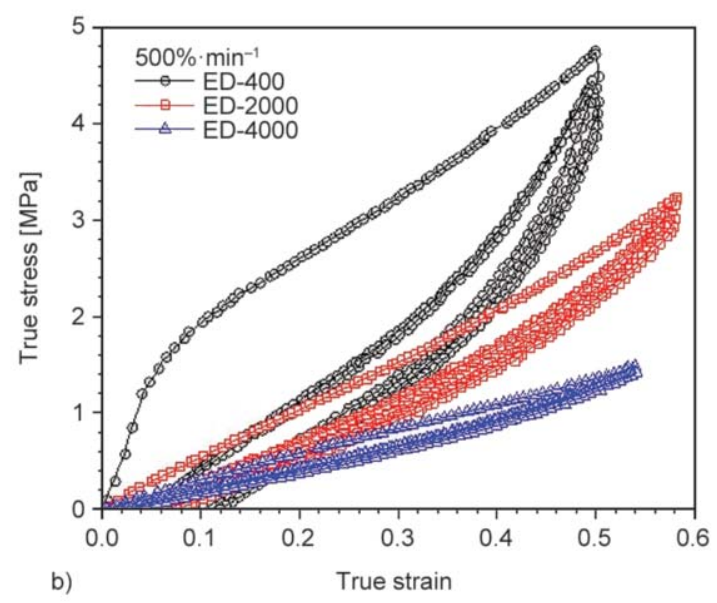

Figure 3. Uniaxial tensile test experiments for the three PU elastomers ED-400 (black circles), ED-2000 (red squares), and ED-4000 (blue triangles) during three loading-unloading cycles up to a maximum strain value of $\varepsilon_{\max }=0.5-0.6$, at the strain rate of a) $100 \% \cdot \mathrm{min}^{-1}$, and b) $500 \% \cdot \mathrm{min}^{-1}$. 
showed hysteresis with residual strain, and true stresstrue strain curves that stabilized after the second cycle. Since the findings of Mullins and coworker [28-30] that filled rubbers undergo softening (referred to as the 'Mullin's effect'), studies in rubber-like materials are generally considered to undergo a similar behavior
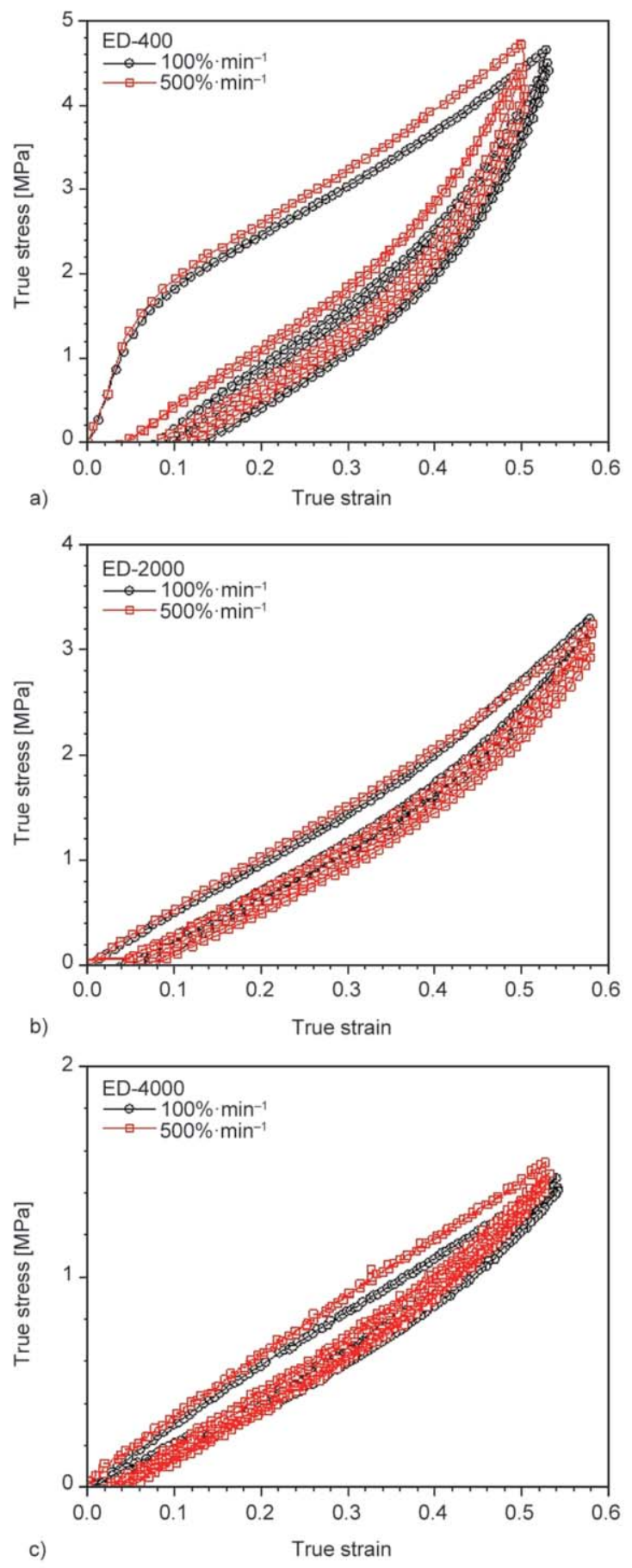

Figure 4. Uniaxial tensile test experiments for the three PU elastomers - a) ED-400, b) ED-2000, and c) ED4000- during three loading-unloading cycles up to a maximum strain value of $\varepsilon_{\max }=0.5-0.6$, at the strain rate of $100 \% \cdot \mathrm{min}^{-1}$ (black circles), and $500 \% \cdot \min ^{-1}$ (red squares).
[27]. All PU materials studied in this work that have a low percentage of hard domains in comparison to soft domains, i.e., ED-2000 and ED-4000, can be considered as elastomeric networks with dispersed particles like for nanocomposites, due to microphase separation - immiscibility - between hard and soft domains. Moreover, this segregation allows hydrogen bond formation and, as a consequence, mechanically reinforces the material. When hydrogen bonds are mechanically or thermally broken, the domains are mixed up, and the material behaves like a conventional elastomer such as silicone networks.

In this work, consecutive uniaxial loading-unloading cycles are performed in the same sample, where each cycle reaches a higher level of strain compared to the previous one. The result of this test is shown in Figure 5. When a new loading cycle starts, the material softens until it reaches the maximum strain level of the previous cycle. This softening should be understood as follows: if we compare the loading part of the curve for two consecutive cycles, the stress value at an equal strain is always lower for the second cycle until the maximum level of strain for the first cycle is reached. From this point forward, the material behaves as loading further from the first cycle. The same behavior is observed in the following cycles for the three PU materials studied in this work. Thus, at this strain rate value, PU networks didn't have enough time to relax and recover a similar polymer conformation with the corresponding retractive force pulling the load cell.

Figure $5 \mathrm{a}-5 \mathrm{c}$ shows the softening effect on all crosslinked PU samples. Additionally, the curve resulting from the sum of the loading segment from the first cycle and the loading segments from the second to the fourth cycle, when reaching the maximum strain value of the previous cycle, coincides with the single uniaxial tension test experiment. This behavior reminds the one firstly observed by Mullin [31].

A significant change in the stress relaxation behavior of the three PU materials is observed in Figure 5d during the uniaxial stress relaxation tensile test experiments. It should be noticed that the stress value for the ED- 400 network shows a decrease of $\sigma_{\text {true }} \%=$ 26 and $27 \%$ of the total stress prior the constant strain step for the first and second relaxation step after $60 \mathrm{~s}$, respectively, while for the ED-2000 and ED-4000 samples the decrease in the stress value were of $\sigma_{\text {true }} \%=10$ and $14 \%$ and of $\sigma_{\text {true }} \%=11$ and 
$12 \%$, respectively. These results confirm the idea that the network with shorter segmental length, ED-400,
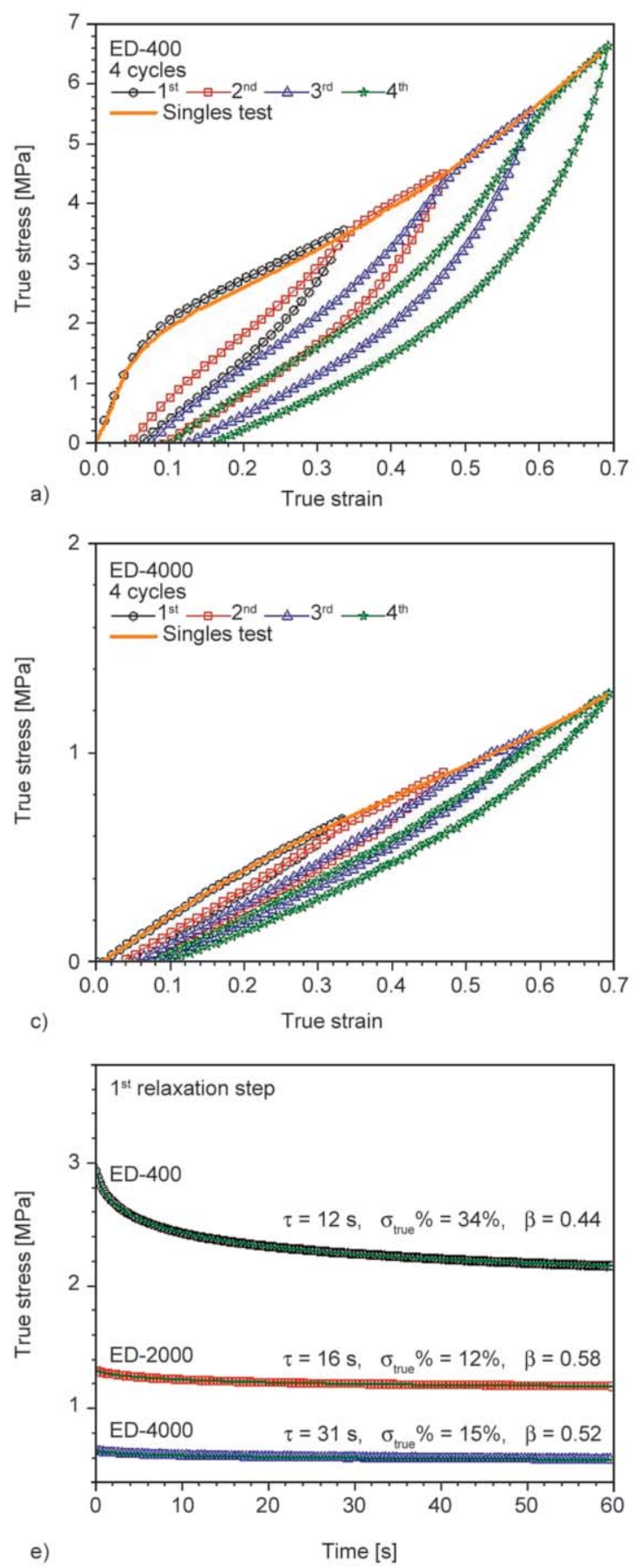

relaxes faster than the longest polymer segments, ED-2000 and ED-4000.
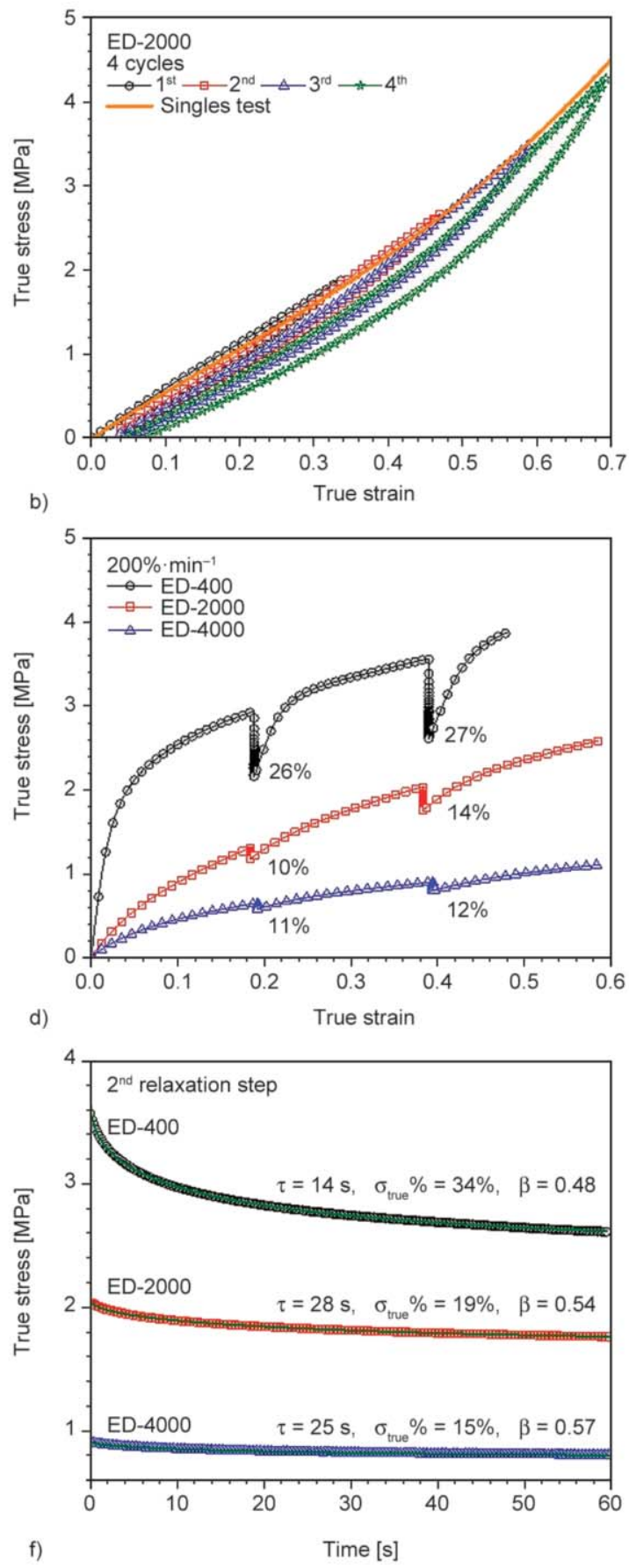

Figure 5. Uniaxial tensile test experiments with four consecutive-extended loading-unloading cycles (black circles, red squares, blue triangles and green stars) and a single loading test for the three PU elastomers (thick orange line) a) ED-400, b) ED-2000, and c) ED-4000 - during four consecutive loading-unloading cycles, at the strain rate of $100 \% \cdot \mathrm{min}^{-1}$. d) Uniaxial stress relaxation tensile test experiments at the cross-head speed of $200 \% \cdot \mathrm{min}^{-1}$ and two stress relaxation steps at $\varepsilon=0.2$ and $\varepsilon=0.4$, for the PU samples ED-400 (black circles), ED-2000 (red squares), and ED-4000 (blue triangles). The values in each curve correspond to the stress value decrease of the total stress prior the constant strain step $\left[\sigma_{\text {true }} \%=1-\sigma_{\text {true }}(60 \mathrm{~s}) / \sigma_{\text {true }}(0 \mathrm{~s})\right]$. e) First and f) second relaxation step analysis for the three PU elastomers, where each set of data where fitted with a stretched exponential decay function. On each plot the corresponding life time $(\tau)$, percentage of stress relaxation value $\left[\sigma_{\text {true }} \%=1-\sigma_{\text {true }}(\infty) / \sigma_{\text {true }}(0 \mathrm{~s})\right]$, and exponential factor $(\beta)$ are indicated. 
An in-depth analysis during the relaxation processes was performed by fitting the experimental data with a stretched exponential decay function (Figure 5e and 5f). The lifetime for the sample ED-400 is the shortest, $\tau=12-14 \mathrm{~s}$, when compared to the other two networks, ED-2000 and ED-4000, with values of $\tau=16-28 \mathrm{~s}$ and $\tau=25-31 \mathrm{~s}$, respectively. Again, this is in agreement with the idea that short segmental polymers relax faster at the same macroscopic strain value. Moreover, the stress value decrease for the sample ED-400 $\left(\sigma_{\text {true }} \%=34 \%\right)$ obtained from the fitting function is again higher than for the other two elastomeric networks, which values fall between 12 and $19 \%$ of its initial stress value.

Samples thermally treated showed a different mechanical response based on the hydrogen bond breakage at $c a . T_{\mathrm{HB}}=70^{\circ} \mathrm{C}$, as shown by Sanchez-Ferrer et al. [2, 7] (Figure 6). Without this physical bonding, the hard domains become smaller, and the segmental molecular weight increases, with the corresponding softening of the PU elastomer.

Irrespectively of the chemical composition of the three PU materials, all samples display a softening behavior after being cooled down to room temperature at the highest cooling rate. This can be accounted for by the fact that hydrogen bonds broke at $70^{\circ} \mathrm{C}$, and a higher cooling rate does not give enough time for the reformation of such physical interactions (red curves in Figure 6). On the contrary, when slowly cooled down, hydrogen bonds formation can take place partially (blue curves in Figure 6), and the PU mechanical behavior approaches a similar behavior to the one of the pristine sample (black curves in Figure 6). The ED-400 sample is an exception due to a lack of hydrogen bond reformation and reduced mobility of the short polymer segments [7] for both fast and slow cooling processes.

\subsection{Constitutive behavior of $P U$ materials}

In light of the above observations, the constitutive model capable of capturing moderate strain deformation of the PU networks should account for the following characteristics: i) non-linear strain behavior; ii) hysteresis and iii) stress softening. As a first attempt, the Bergström-Boyce constitutive model with Mullins' damage accumulation [32] (for the BBM model numerical implementation refer to [33]) was evaluated. Using an inverse method optimization procedure [34], the constitutive parameters were
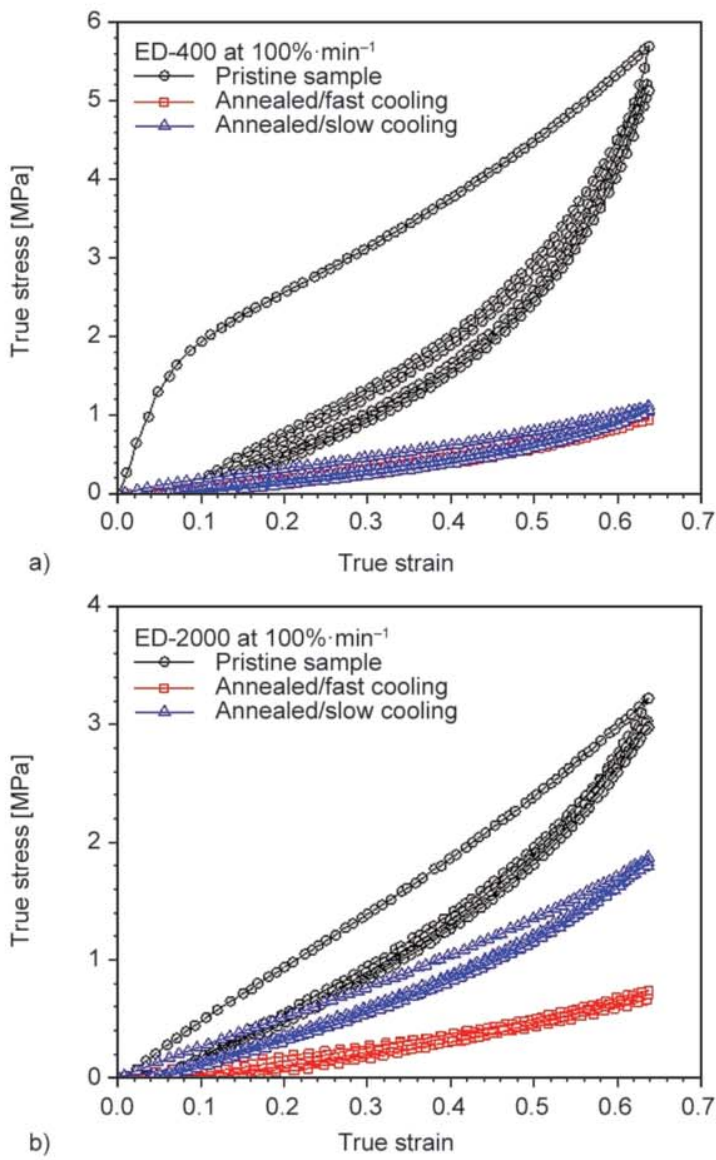

b)

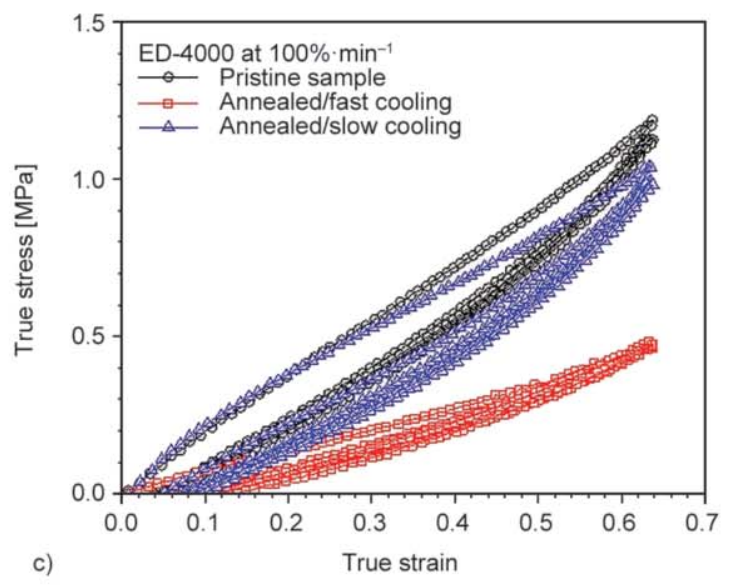

Figure 6. Uniaxial tensile test experiments for the three PU elastomers - a) ED-400, b) ED-2000, and c) ED4000 - during three loading-unloading cycles, at the strain rate of $100 \% \cdot \mathrm{min}^{-1}$, and with different thermal story: pristine sample (black circles), annealed at $70^{\circ} \mathrm{C}$ and slow cooling (blue triangles), and annealed at $70^{\circ} \mathrm{C}$ and fast cooling (red squares).

determined by fitting model simulations to the experimental true stress-true strain monotonic and cyclic tensile curves. For this purpose, the MCalibration commercial software by Veryst Engineering was utilized. The calibration was carried out in a sequence 
scheme and a final tunning of the constitutive parameters was performed with the whole experimental data simultaneously.

It was found that for both the ED-2000 and ED-4000 sample, which show a typical elastomeric response, the viscoelastic constitutive model chosen (BBM) [32], accurately described the experimental data (Figure 7a and 7b). The simulated curves based on the BBM model agree well with the experimental data exhibiting the features of substantial resilience and dissipation, including stretch-induced softening during cyclic tension. On the contrary, for the ED-400 sample, which displays a mixed mechanical response with 'elastomeric' and 'glass-like' features, the BBM model was not able to accurately capture the material response (Figure 7c).

Accordingly, a different model that considers the glass-like response of the hard and soft domains was implemented in order to match the mechanical response for the ED-400 sample. We considered using the Three Network (TN) model [33] (for the TN model numerical implementation refer to [35]), which
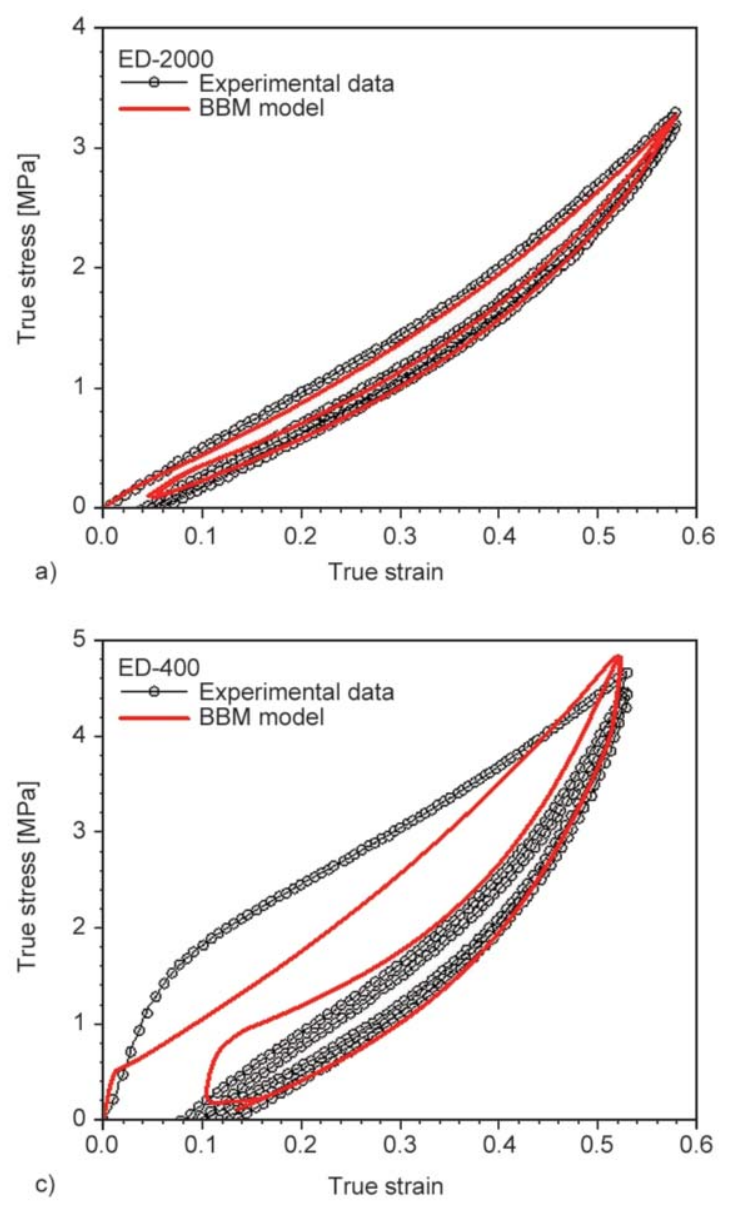

was initially designed to represent the mechanical behavior of thermoplastic materials. The mathematical equations are represented by three molecular networks acting in parallel, (refer to [32] for details on the constitutive model). Network A and B capture the initial viscoplastic response governed by two activation mechanisms in accordance with the amorphous and semicrystalline domains. Network C controls the large strain mechanical response by means of the entropic resistance. These features allow the constitutive material model to consider the elastic and plastic deformation typical for thermoplastic materials. The temperature-dependent parameters are not included in this work, thus they were not considered when obtaining the model parameters. Some of the most important mechanical characteristics observed for the sample ED- 400 are the fact that i) it is weakly strain-rate dependent, ii) linearly elastic for strain values lower than 0.03 , and iii) with a clear transition at a strain values between 0.05 and 0.1 similar to the one observed in thermoplastic polymers. For the reasons mentioned above, the TN model was selected for
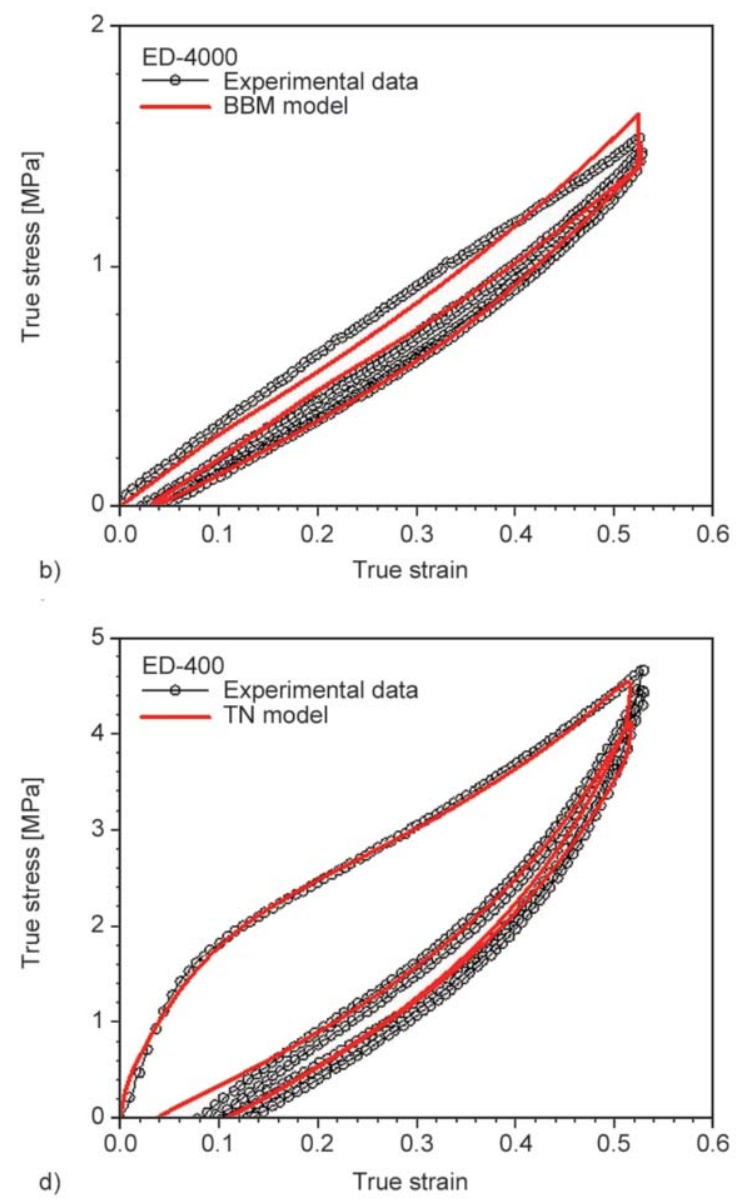

Figure 7. Experimental results (black circles) and constitutive models (red curves) for the three crosslinked PU samples. BBM model: a) ED-2000, b) ED-4000, and c) ED-400; TN model: d) ED-400. 
fitting the experimental data, and the results showed an accurate fit that describes the mechanical behavior of this material (Figure 7d). Elastomers usually do not show the transition of the elastic and viscoplastic part of the stress-strain curve, but it was found for the sample ED-400 due to its high crosslinking density.

\section{Conclusions}

The mechanical behavior of three crosslinked PU elastomers obtained via sol-gel chemistry with different architecture was assessed as a function of the segmental molecular weight of the soft segments. Both the PU ED-2000 and ED-4000 with a high volume fraction of soft domains showed an elastomeric behavior due to the low percentage of hard domains (crosslinking sites), which mainly act as reinforcement as for nanocomposites, whereas the PU ED400 displayed a glass-like behavior due to a higher volume fraction of hard domains.

Mechanical test experiments up to strain values of $\varepsilon_{\max }=0.5-0.6$ were carried out using a uniaxial testing machine, varying the crosshead speed and performing three loading-unloading cycles. These tests showed some inelastic features of rubbers such as hysteresis, softening and the presence of residual strain. It was shown that a widely adopted constitutive model to describe non-linear hyperelastic behavior of elastomers, i.e., the Bergström-Boyce, with the addition of the Mullins' effect model (BBM), was accurate in order to describe the viscoelastic timedependent mechanical response obtained for both ED-2000 and ED-4000 samples. On the other hand, the Three Network model (TN) could capture the viscoplastic behavior for the ED-400 'glass-like' sample with a yield event.

Additionally, this work evidences the constitutive model (TN) potential, which was initially created to describe the mechanical behavior of thermoplastic materials and able to describe the viscoelastic behavior of a nanocomposite with dispersed soft domains embedded into a glassy network. Moreover, it is a robust model that does not present major inconvenient when implemented numerically and has the advantages of being user-friendly, commercial and standard. Moreover, future work could also incorporate the effect of temperature by adding a temperature-dependent power-law viscoplastic flow element to the model and performing uniaxial test experiments at a wide range of temperatures.

\section{Acknowledgements}

We thank the financial support from CONICET, MinCyT, Universidad Nacional de Mar del Plata and PICT 2016-0302.

\section{References}

[1] Primeaux D. J.: Polyurea elastomer technology: History, chemistry and basic formulating techniques. Primeaux Associates LLC, Elgin (2004).

[2] Sánchez-Ferrer A., Rogez D., Martinoty P.: Influence of the degree of polymerisation and of the architecture on the elastic properties of new polyurea elastomers. RSC Advances, 5, 6758-6770 (2015). https://doi.org/10.1039/C4RA09879J

[3] Bradley S.: The chemistry and properties of the medical-grade silicones. Journal of Macromolecular Science: Part A: Chemistry, 4, 529-544 (1970). https://doi.org/10.1080/00222337008074361

[4] Weathersby P. K., Kolobow T., Stool E. W.: Relative thrombogenicity of polydimethylsiloxane and silicone rubber constituents. Journal of Biomedical Materials Research, 9, 561-568 (1975). https://doi.org/10.1002/jbm.820090604

[5] Boretos J. W., Pierce W. S.: Segmented polyurethane: A new elastomer for biomedical applications. Science, 158, 1481-1482 (1967).

https://doi.org/10.1126/science.158.3807.1481

[6] Hergenrother R. W., Yu X-H., Cooper S. L.: Blood-contacting properties of polydimethylsiloxane polyureaurethanes. Biomaterials, 15, 635-640 (1994). https://doi.org/10.1016/0142-9612(94)90215-1

[7] Sánchez Ferrer A., Rogez D., Martinoty P.: Synthesis and characterization of new polyurea elastomers by sol/gel chemistry. Macromolecular Chemistry and Physics, 211, 1712-1721 (2010). https://doi.org/10.1002/macp.201000117

[8] Buckwalter D. J., Dennis J. M., Long T. E.: Amide-containing segmented copolymers. Progress in Polymer Science, 45, 1-22 (2015).

https://doi.org/10.1016/j.progpolymsci.2014.11.003

[9] Roland C. M., Twigg J. N., Vu Y., Mott P. H.: High strain rate mechanical behavior of polyurea. Polymer, 48, 574-578 (2007).

https://doi.org/10.1016/j.polymer.2006.11.051

[10] Sarva S. S., Deschanel S., Boyce M. C., Chen W.: Stress-strain behavior of a polyurea and a polyurethane from low to high strain rates. Polymer, 48, 2208-2213 (2007).

https://doi.org/10.1016/j.polymer.2007.02.058

[11] Grujicic M., He T., Pandurangan B., Svingala F. R., Settles G. S., Hargather M. J.: Experimental characterization and material-model development for microphase-segregated polyurea: An overview. Journal of Materials Engineering and Performance, 21, 2-16 (2012). https://doi.org/10.1007/s11665-011-9875-6 
[12] Bai Y., Liu C., Huang G., Li W., Feng S.: A hyper-viscoelastic constitutive model for polyurea under uniaxial compressive loading. Polymers, 8, 133/1-133/17 (2016). https://doi.org/10.3390/polym8040133

[13] Reppel T., Dally T., Weinberg K.: On the elastic modeling of highly extensible polyurea. Technische Mechanik, 33, 19-33 (2013).

[14] Shim J.: Finite strain behavior of polyurea for a wide range of strain rates. Massachusetts Institute of Technology, Massachusetts (2010).

[15] Cho H., Rinaldi R. G., Boyce M. C.: Constitutive modeling of the rate-dependent resilient and dissipative large deformation behavior of a segmented copolymer polyurea. Soft Matter, 9, 6319-6330 (2013). https://doi.org/10.1039/C3SM27125K

[16] Yilgor I., Eynur T., Bilgin S., Yilgor E., Wilkes G. L.: Influence of soft segment molecular weight on the mechanical hysteresis and set behavior of silicone-urea copolymers with low hard segment contents. Polymer, 52, 266-274 (2011). https://doi.org/10.1016/j.polymer.2010.11.040

[17] Ertem S. P., Yilgor E., Kosak C., Wilkes G. L., Zhang M., Yilgor I.: Effect of soft segment molecular weight on tensile properties of poly(propylene oxide) based polyurethaneureas. Polymer, 53, 4614-4622 (2012). https://doi.org/10.1016/j.polymer.2012.08.020

[18] Lev Y., Faye A., Volokh K. Y.: Experimental study of the effect of temperature on strength and extensibility of rubberlike materials. Experimental Mechanics, 58, 847-858 (2018). https://doi.org/10.1007/s11340-018-0374-7

[19] Jerrams S., Murphy N., Hanley J.: The significance of equi-biaxial bubble inflation in determining elastomeric fatigue properties. in 'Advanced elastomers, Technology, properties and applications' (ed.: Boczkowska A.) Intech Rijeka, 379-381 (2012).

https://doi.org/10.5772/50099

[20] Feng W. W., Hallquist J. O.: On constitutive equations for elastomers and elastomeric foams. in 'The 4th European LS-DYNA Conference, Ulm, Germany’ D-II-15 (2003).

[21] Mooney M.: A theory of large elastic deformation. Journal of Applied Physics, 11, 582-592 (1940). https://doi.org/10.1063/1.1712836

[22] Ogden R. W.: Non-linear elastic deformations. Dover Publications, Mineola (1997).

[23] Sebastián N., Contal C., Sánchez-Ferrer A., Pieruccini M.: Interplay between structure and relaxation in polyurea networks: The point of view from a novel method of cooperativity analysis of dielectric response. Soft Matter, 14, 7839-7849 (2018).

https://doi.org/10.1039/C8SM01113C
[24] Reinecker M., Soprunyuk V., Fally M., Sánchez-Ferrer A., Schranz W.: Two glass transitions of polyurea networks: Effect of the segmental molecular weight. Soft Matter, 10, 5729-5738 (2014).

https://doi.org/10.1039/C4SM00979G

[25] Sánchez-Ferrer A., Finkelmann H.: Thermal and mechanical properties of new main-chain liquid-crystalline elastomers. Molecular Crystals and Liquid Crystals, 508, 348-710 (2009).

https://doi.org/10.1080/15421400903065861

[26] Sánchez Ferrer A., Finkelmann H.: Polydomain-monodomain orientational process in smectic-C main-chain liquid-crystalline elastomers. Macromolecular Rapid Communications, 32, 309-315 (2011). https://doi.org/10.1002/marc.201000590

[27] Qi H. J., Boyce M. C.: Stress-strain behavior of thermoplastic polyurethanes. Mechanics of Materials, 37, 817-839 (2005).

https://doi.org/10.1016/j.mechmat.2004.08.001

[28] Mullins L.: Effect of stretching on the properties of rubber. Rubber Chemistry and Technology, 21, 281-300 (1948).

https://doi.org/10.5254/1.3546914

[29] Mullins L., Tobin N. R.: Theoretical model for the elastic behavior of filler-reinforced vulcanized rubbers. Rubber Chemistry and Technology, 30, 555-571 (1957). https://doi.org/10.5254/1.3542705

[30] Mullins L.: Softening of rubber by deformation. Rubber Chemistry and Technology, 42, 339-362 (1969). https://doi.org/10.5254/1.3539210

[31] Diani J., Fayolle B., Gilormini P: A review on the Mullins effect. European Polymer Journal, 45, 601-612 (2009). https://doi.org/10.1016/j.eurpolymj.2008.11.017

[32] Bergström J. S., Boyce M. C.: Constitutive modeling of the large strain time-dependent behavior of elastomers. Journal of the Mechanics and Physics of Solids, 46, 931-954 (1998). https://doi.org/10.1016/S0022-5096(97)00075-6

[33] Bergström J. S., Bischoff J. E.: An advanced thermomechanical constitutive model for UHMWPE. The International Journal of Structural Changes in Solids, 2, 31-39 (2010).

[34] Polanco Loria M., Daiyan H., Grytten F.: Material parameters identification: An inverse modeling methodology applicable for thermoplastic materials. Polymer Engineering and Science, 52, 438-448 (2012). https://doi.org/10.1002/pen.22102

[35] Veryst Engineering: PolyUMod user's manual. Veryst Engineering, Needham (2009). 